\title{
Disfunción del conducto faringotimpánico. EI ETDQ-7 como herramienta para el diagnóstico clínico
}

\section{Pharyngothympanic tube dysfunction. The ETDQ-7 as a tool for clinical diagnosis}

\author{
Elvira Cortese $\mathbf{S}^{1,2}$, Rocío Cerda $\mathbf{V}^{2}$, Constanza Nahuelpan $\mathbf{P}^{2}$, y Marlene Silva G².
}

\begin{abstract}
RESUMEN
En la actualidad se hace referencia en la bibliografía a una gran variedad de métodos para evaluar la disfunción del conducto faringotimpánico (DCFT), la gran mayoría de ellos requiere de instrumental de alta complejidad y personal altamente capacitado. Pese a lo mencionado, ninguno de dichos métodos ha sido validado como herramienta gold standard para el diagnóstico de esta patología. En este contexto, se presenta una herramienta complementaria para el diagnóstico de la DCFT, el Eustachian Tube Dysfunction Questionnaire -7 ítemes (ETDQ-7); cuestionario autoadministrado que considera 7 preguntas en base a una escala de Likert, fue creado para evaluar la sintomatología asociada a la disfunción del conducto con una puntuación que va desde lo más leve (1 punto) a lo más grave (7 puntos) de acuerdo con los síntomas percibidos, y posee un puntaje máximo de 49 puntos, correspondiente a una disfunción severa. La presente revisión tiene como objetivo buscar, organizar, clasificar y describir la información disponible en publicaciones científicas indexadas sobre el ETDQ-7 como herramienta para el diagnóstico clínico complementario de la DCFT. Se incluyeron 11 artículos científicos que hacen referencia al uso del ETDQ-7 en diferentes países. Los resultados recabados sugieren que el ETDQ-7 sería una valiosa herramienta, de alta sensibilidad, especificidad y validez clínica, que permitiría discriminar la presencia de la DCFT. No se encontró evidencia respecto a la aplicación o validación de este cuestionario en nuestro país.
\end{abstract}

Palabras clave: ETDQ-7, disfunción del conducto faringotimpánico, trompa de Eustaquio, disfunción obstructiva, disfunción patulosa.

\begin{abstract}
At present, great variety of methods are described to evaluate the dysfunction of the pharyngotympanic duct (DCFT), the vast majority of them require highly complex instruments and highly trained personnel. Despite this, none of these methods has been validated as the gold standard for the diagnosis of this pathology. In this context, a complementary tool for the diagnosis of the DCFT is presented. The Eustachian Tube Dysfunction Questionnaire-7 items (ETDQ-7), a self-administered questionnaire that in-
\end{abstract}

\footnotetext{
1 Centro de Atención Fonoaudiológica, Universidad de Valparaíso, Chile.

2 Escuela de Fonoaudiología, Facultad de Medicina, Universidad de Valparaíso. Valparaíso, Chile.

Los autores declaran no tener conflictos de interés.
}

Recibido el 10 de mayo de 2019. Aceptado el 29 de mayo de 2019. 
cludes 7 questions based on a Likert scale, was created to evaluate the symptomatology associated with the dysfunction of the duct. It is scored ranging from the mildest symptomatology (1 point) to the most severe (7 points), according to the perceived symptoms, with a maximum score of 49 points, corresponding to a severe dysfunction. The present review aims to search, organize, classify and describe the available information in scientific indexed journals about the ETDQ-7 as a tool for complementary diagnosis of the DCFT. Eleven scientific articles were included, referred to the use of ETDQ-7 in different countries, describing it as a valid tool which allows to discriminate the presence of a DCFT.

Key words: ETDQ-7, eustachian tube dysfunction questionnaire, pharyngotympanic tube dysfunction, obstructive eustachian tube, patulous eustachian tube.

\section{INTRODUCCIÓN}

La disfunción del conducto faringotimpánico (DCFT) es una patología muy común en la clínica otorrinolaringológica, especialmente en niños ${ }^{1-3}$. El diagnóstico diferencial de la DCFT se realiza mediante una amplia batería de pruebas, tanto subjetivas como objetivas, cuyos resultados se complementan para establecer el diagnóstico de la DCFT5. Entre las pruebas instrumentales más solicitadas en la clínica por los especialistas se encuentra la evaluación timpanométrica, específicamente, la evaluación de la permeabilidad tubaria 0 prueba de función tubaria ${ }^{1,2,6-9}$. Actualmente, existe información escasa y controversial acerca del diagnóstico y evaluación de la disfunción del conducto faringotimpánico ${ }^{10,11}$. Lo anterior es aún más evidente, cuando se busca información específica respecto a la utilidad, uso y aplicación de cuestionarios de autovaloración de síntomas o de resultados informados por el paciente como el Eustachian Tube Dysfunction Questionnaire-7(ETDQ-7), para el diagnóstico clínico de la disfunción del conducto faringotimpánico. Tomando en cuenta que dicha disfunción es un cuadro de elevada prevalencia en la clínica ${ }^{1,11}$ y no existe suficiente evidencia que avale el uso de una metodología por sobre el resto como herramienta gold standard para establecer un diagnóstico certero ${ }^{11}$ surge la necesidad de investigar respecto a la disponibilidad de instrumentos alternativos, de bajo costo y fácil aplicación, cuya sensibilidad y especificidad los conviertan en herramientas útiles en la práctica clínica, para el diagnóstico de la DCFT.

La presente revisión bibliográfica se centra en un método complementario de evaluación subjetiva, en el área de la clínica otorrinolaringológica:
El Eustachian Tube Dysfunction Questionnaire-7 (ETDQ-7); (Tabla 1) cuestionario autoadministrado, elaborado en base a una escala de Likert que incluye 7 preguntas mediante las que se solicita al encuestado indicar nivel de afectación o severidad percibida para cada declaración establecida por ítem. En cada pregunta, las posibilidades de puntuación van desde lo más leve (1 punto) a lo más grave (7 puntos) de acuerdo con los síntomas percibidos. La prueba tiene un máximo posible de 49 puntos, correspondiente a una disfunción severa. EI ETDQ-7 fue diseñado para apoyar el diagnóstico clínico de la disfunción del conducto faringotimpánico; estructura crucial en el drenaje, ventilación y protección del oído medio. Una alteración en el funcionamiento de dicho conducto puede generar variadas consecuencias, enmarcadas en el cuadro de "disfunción del conducto faringotimpánico", en adelante DCFT'12-15. La DCFT puede presentarse asociada a múltiples síntomas clínicos, como sensación de oído tapado u obstruido, dolor 0 presión en los oídos, entre otros ${ }^{11,15-19}$. La presente revisión tiene como objetivo buscar, organizar, clasificar y describir la información disponible en publicaciones científicas indexadas, tanto nacionales como internacionales, sobre el ETDQ-7 como herramienta complementaria para el diagnóstico clínico de la DCFT.

En la actualidad, se reconoce que el impacto que una enfermedad genera en la persona no puede ser descrita en su totalidad por medidas objetivas de la salud como resultado de un examen en particular. Tener en cuenta otros factores, como la subjetividad del propio paciente, se considera imprescindible a la hora de evaluar de forma global el estado de salud de las personas ${ }^{5,8}$. Esta nueva 
perspectiva intenta calificar la enfermedad 0 disfuncionalidad desde el punto de vista del paciente. Los cuestionarios en salud cumplen una función facilitadora en la evaluación, permitiendo que el paciente exprese y/o cuantifique sus síntomas respecto a una molestia en particular, razón por la cual, su uso se ha masificado en el ámbito clínico ${ }^{20}$. Entre algunos beneficios del uso de escalas, cuestionarios, test e índices en salud, se encuentran: ayudar al diagnóstico, apoyar el tratamiento y permitir una comunicación rápida y eficaz con otros profesionales ${ }^{21}$.

En la elección de una determinada pauta o cuestionario, es fundamental evaluar sus indicadores de validez y confiabilidad. Estos denotan la utilidad científica de la escala, en otras palabras, en qué magnitud se ajusta lo observado a lo que se pretende medir. Por su parte, la confiabilidad además demuestra su precisión a la hora de valorar, poniendo a prueba su reproducibilidad en posteriores medidas 0 por distintos observadores ${ }^{8,21}$. Un cuestionario que cumple con dichos requisitos puede ser de ayuda en el diagnóstico, ya que complementa la anamnesis y observación clínica, permitiendo llegar a un diagnóstico de manera más expedita y con reducciones importantes en costos asociados a exámenes 0 procedimientos instrumentales ${ }^{21}$.

Las encuestas sobre la salud se clasifican en genéricas y específicas o relativas a una patología concreta. Las encuestas específicas, como bien dice su nombre, están hechas para determinar la presencia de una patología o grupos de patologías²2. Algunos de estos cuestionarios específicos se presentan como excelentes alternativas para esclarecer el diagnóstico clínico en cuadros de difícil consenso, como ha sido el estudio de la DCFT. Un ejemplo de este tipo de cuestionarios es el ETDQ-7 ${ }^{13,22}$, que tiene por objetivo evaluar la DCFT. Este cuestionario se desarrolló utilizando una metodología de encuesta estándar, fue completado por un grupo de 50 pacientes adultos consecutivos diagnosticados con DCFT y 25 pacientes sin DCFT que sirvieron como grupo de control. La timpanometría se usó como criterio estándar para distinguir los dos grupos. Un subconjunto de encuestados repitió el ETDQ-7 en un momento determinado 4 semanas después. Finalmente, el cuestionario quedó conformado por siete preguntas cerradas, en base a una escala de Likert, que recopila información de severidad del 1 al 7 en relación con los síntomas típicos de la DCFT. El puntaje máximo que correlaciona con una disfunción severa del conducto faringotimpánico, corresponde a 49 puntos $^{13}$.

El objetivo general de este estudio fue describir y sistematizar la información existente a nivel nacional e internacional (en publicaciones científicas indexadas), respecto al ETDQ-7, su uso y aplicación como herramienta para el diagnóstico

\section{Tabla 1. Cuestionario para la disfunción de la trompa de Eustaquio: Eustachian Tube Dysfunction Patient Questionnaire (ETDQ-7)}

\begin{tabular}{|c|c|c|c|c|c|c|c|c|}
\hline Afectación & \multicolumn{2}{|c|}{ Leve } & \multicolumn{3}{|c|}{ Moderada } & \multicolumn{2}{|c|}{ Severa } & Puntaje \\
\hline 1 ¿Presión en los oídos? & 1 & 2 & 3 & 4 & 5 & 6 & 7 & \\
\hline 2 ¿Dolor de oídos? & 1 & 2 & 3 & 4 & 5 & 6 & 7 & \\
\hline 3 ¿Sensación de oído tapado o de escuchar "bajo el agua"? & 1 & 2 & 3 & 4 & 5 & 6 & 7 & \\
\hline 4 ¿Problemas de oído asociados a resfríos o sinusitis? & 1 & 2 & 3 & 4 & 5 & 6 & 7 & \\
\hline 5 ¿Ruidos como crujidos o chasquidos en los oídos? & 1 & 2 & 3 & 4 & 5 & 6 & 7 & \\
\hline 6 ¿Zumbido en los oídos? & 1 & 2 & 3 & 4 & 5 & 6 & 7 & \\
\hline 7 ¿Sensación de audición débil o "apagada"? & & & 3 & 4 & 5 & 6 & 7 & \\
\hline C. ¿Siente las molestias en uno u ambos oídos? & \multicolumn{2}{|c|}{ Izquierda } & \multicolumn{3}{|c|}{ Derecha } & \multicolumn{2}{|c|}{ Ambos } & \\
\hline
\end{tabular}

Nota. Adaptación al español del cuestionario Eustachian Tube Dysfunction Patient Questionnaire (ETDQ-7). Copyright 2012 by McCoul ED, Anand VK and Christos PJ. Fuente: McCoul ED., Anand VK., Christos PJ. (2012) Validating the clinical assessment of eustachian tube dysfunction: The Eustachian Tube Dysfunction Questionnaire (ETDQ-7). Laryngoscope 122 (5), pp 1137-41. Reproducido con permiso 0 Dominio Público. 
complementario de la disfunción del conducto faringotimpánico. El trabajo se basó en un enfoque cualitativo con alcance descriptivo y diseño de tipo documental. Se trabajó con un tipo de muestreo no probabilístico, que permitió la selección del objeto de estudio a investigar bajo el criterio de información confiable y actualizada. Para determinar el tamaño de la muestra en este estudio se utilizó el criterio de saturación de la información. Las unidades de información utilizadas en este estudio corresponden a artículos de revistas científicas indexadas en EBSCO, ClinicalKey, Scielo (Conicyt) y Pubmed, y literatura clásica que cumplían con los requisitos de inclusión preestablecidos.

Se realizó una búsqueda bibliográfica entre marzo y noviembre de 2018, desde motores de búsqueda seguros como lo son EBSCO, ClinicalKey, SciELO (Conicyt) y Pubmed. Se consideraron todos los artículos de no más de 5 años de antigüedad, sin restricción respecto al país de origen o el idioma. Se incluyó en la redacción final toda la información relevante de 11 artículos científicos

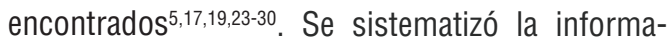
ción de todos los artículos, entre los que predominó el idioma inglés. El rango etario de población abordado fue entre los 11 a 89 años.

El ETDQ-7 elaborado por McCoul y cols en 2012, ha sido traducido, adaptado y validado en distintos países del mundo: Inglaterra, Estados Unidos, Alemania, Turquía, Bélgica, Israel y Brasil. Los estudios han demostrado que dicho cuestionario es un instrumento que presenta buenos indicadores estadísticos para la detección de la DCFT, lo que lo convierte en una herramienta confiable. En el desarrollo de esta revisión se encontraron aproximadamente 30 artículos de divulgación científica, cuyo tema a tratar era la DCFT y métodos de evaluación que no incluían el cuestionario. La muestra consideró artículos que se centraban en el estudio del ETDQ-7 o lo incluían como instrumento de evaluación $n^{5,17,19,23-30}$. No se encontró ningún artículo en Chile que hiciera referencia al uso del ETDQ-7 como apoyo para el diagnóstico de la DCFT. En Tabla 2 se presenta un resumen de las publicaciones que conformaron la muestra. Se presenta el título del artículo, los autores, el año de publicación, la técnica de recolección utilizada en el estudio y de manera general, se exponen los principales resultados y conclusiones presentadas por los autores, que se describen de forma sucinta a continuación.

En cuanto a la validez discriminante del ETDQ7, un estudio llevado a cabo en Bélgica por el grupo de Van Roeyen, Van de Heyning \& Van Rompaey (2016), concluyó que dicho cuestionario puede discriminar entre pacientes con DCFT inducida por cambios de presión atmosférica y controles sanos y que responde al cambio en pacientes con DCFT inducida por cambios de presión atmosférica que han sido sometidos a tuboplastía por dilatación de balón $(\mathrm{TDB})^{30}$. Asimismo, otro estudio realizado por el grupo de Han y cols en 2017, comparó la aplicación del ETDQ-7 con la evaluación objetiva mediante Endoscopía con Video en Cámara Lenta (EVCL), concluyendo que ambas herramientas proporcionan información sobre el estado preoperatorio de la DCFT, midiendo los cambios estructurales dinámicos del CFT en combinación con otras pruebas de diagnóstico ${ }^{5}$.

Por otro lado, el estudio presentado por el grupo de Van Roeyen y cols, (2015), buscó determinar el valor y el poder discriminativo del ETDQ-7 en pacientes con DCFT-OC y DCFT-P29, concluyendo que el instrumento no puede discriminar entre ambas patologías, sin embargo, su fiabilidad es adecuada cuando se refiere a éstas y se confirma su excelente validez discriminante para un grupo de personas sin DCFT. En cuanto a la validación del ETDQ-7 en otros países, tanto en Turquía, como en Israel, Brasil y Alemania se realizaron estudios con este fin. En todos los casos, se concluyó que el cuestionario de siete elementos es de fácil aplicación, y ha demostrado un alto nivel de validez y confiabilidad para la evaluación de los síntomas relacionados con la DCFT. Adicionalmente, se concluye que el ETDQ-7 presenta una excelente capacidad de discriminación, con valores de sensibilidad y especificidad cercanos al $100 \%$. Su resultado respalda antecedentes específicos de sospecha de DCFT-OC y se puede utilizar como una herramienta adicional para el seguimiento después del tratamiento quirúrgico, concluyendo que sus resultados son reproducibles y es un instrumento válido para evaluar la DCFT ${ }^{17,24-26}$. Cabe destacar que la especificidad del ETDQ-7 baja cuando se aplica en pacientes con otras patologías. Lo anterior ha sido reportado en estudios que han aplicado el cuestionario a pacientes con antecedentes de pérdida de audición 
Tabla 2 Artículos científicos indexados referentes a ETDQ-7

\begin{tabular}{|c|c|c|c|}
\hline Título & Autor y fecha de publicación & Metodología & Principales resultados \\
\hline $\begin{array}{l}\text { Beurteilung der } \\
\text { chronisch-obstruktiven } \\
\text { Tubenfunktionsstörung, } \\
\text { evaluation des Eustachian } \\
\text { Tube Dysfunction Ques- } \\
\text { tionnaire in der deutschen } \\
\text { Übersetzung }\end{array}$ & $\begin{array}{l}\text { Schröder S, Lehmann M, } \\
\text { Sudhoff H \& Ebmeyer J. } \\
\text { (2013) }\end{array}$ & $\begin{array}{l}\text { Se realizó la adaptación del ETDQ-7 en } \\
\text { su versión original, al idioma. Se aplicó } \\
\text { el cuestionario adaptado, a un grupo } \\
\text { control (GC) conformado por } 100 \text { adul- } \\
\text { tos sanos, cuyas edades fluctuaban } \\
\text { entre } 17 \text { y } 84 \text { años y al grupo estudio } \\
\text { (GE), conformado por } 43 \text { pacientes con } \\
\text { DCFT-OC, cuyas edades fluctuaban en- } \\
\text { tre } 14 \text { y } 79 \text { años. La respuesta al cues- } \\
\text { tionario fue voluntaria y anónima. }\end{array}$ & $\begin{array}{l}\text { Los resultados del cuestionario ETDQ- } \\
7 \text { en inglés fueron confirmados por } \\
\text { los exámenes de la versión alemana } \\
\text { del cuestionario. La puntuación media } \\
\text { ETDQ- } 7 \text { fue de } 8,67 \text { en los sujetos sa- } \\
\text { nos y de } 24,7 \text { una sensibilidad de } 90,7 \% \\
\text { y especificidad de } 95 \% \text {. El área bajo la } \\
\text { curva en el análisis ROC fue de } 98,8 \% \text { en } \\
\text { los pacientes con disfunción obstructiva } \\
\text { crónica del CFT. }\end{array}$ \\
\hline $\begin{array}{l}\text { A novel diagnostic tool } \\
\text { for chronic obstructive } \\
\text { Eustachian Tube Dys- } \\
\text { function - The Eustachian } \\
\text { Tube score }\end{array}$ & $\begin{array}{l}\text { Schroeder S, Lehmann M, } \\
\text { Sauzet 0, Ebmeyer J. \& } \\
\text { Sudhoff H (2014) }\end{array}$ & $\begin{array}{l}\text { Estudio clínico combinado, prospectivo } \\
\text { y retrospectivo en centro de referencia } \\
\text { terciario. Se realizó la validación de un } \\
\text { nuevo instrumento para evaluación de } \\
\text { la DCFT, el ETS-7. Se comparan re- } \\
\text { sultados obtenidos mediante historia } \\
\text { clinica, timpanometría, audiometría, } \\
\text { tubomanometría y ETDQ-7. } \\
\text { GC conformado por } 215 \text { sujetos sa- } \\
\text { nos, cuya edad fluctuaba entre los } 16 \\
\text { y } 89 \text { años, mientras que el GE estaba } \\
\text { compuesto por } 171 \text { de sujetos de edad } \\
\text { entre } 14 \text { años y } 81 \text { años con DCFT-OC. }\end{array}$ & $\begin{array}{l}\text { El ETDQ-7 es un instrumento válido para } \\
\text { evaluar la función del CFT, por lo tanto } \\
\text { es utilizado como prueba estándar en la } \\
\text { evaluación del nuevo cuestionario pro- } \\
\text { puesto. } \\
\text { Los autores identifican un punto de corte } \\
\text { de } 7 \text { puntos para la DCFT-OC, con una } \\
\text { sensibilidad del } 96 \% \text { y una especificidad } \\
\text { del } 96 \% \text {, utilizando el nuevo ETS-7. }\end{array}$ \\
\hline $\begin{array}{l}\text { Value and Discriminative } \\
\text { Power of the Seven-Item } \\
\text { Eustachian Tube Dys- } \\
\text { function Questionnaire }\end{array}$ & $\begin{array}{l}\text { Van Roeyen S, Van de } \\
\text { Heyning P \& Van Rompaey } \\
\text { V. (2015) }\end{array}$ & $\begin{array}{l}\text { Estudio en el cual participaron } 69 \\
\text { personas; se conformaron } 3 \text { grupos, } \\
\text { el grupo } 1 \text { estaba conformado por } 39 \\
\text { pacientes con DCFT-OC, grupo } 2 \text { por } 8 \\
\text { pacientes con DCFT-P y grupo } 3 \text { por } 22 \\
\text { pacientes sanos. El ETDQ-7 fue aplica- } \\
\text { do a los } 3 \text { grupos. La consistencia in- } \\
\text { terna se evaluó utilizando el coeficiente } \\
\text { de alfa de Cronbach. La característica } \\
\text { operativa del receptor (ROC) se deter- } \\
\text { minó como una medida de precisión. }\end{array}$ & $\begin{array}{l}\text { El ETDQ-7 no puede discriminar entre } \\
\text { DCFT-OC y DCFT-P, sin embargo, se } \\
\text { confirma una excelente validez discrimi- } \\
\text { nante entre sujetos con DCFT y controles } \\
\text { sanos. }\end{array}$ \\
\hline $\begin{array}{l}\text { Eustachian tube } \\
\text { dysfunction: consensus } \\
\text { statement on definition, } \\
\text { types, clinical presen- } \\
\text { tation }\end{array}$ & $\begin{array}{l}\text { Schilder AM, Bhutta C, Butler } \\
\text { C, Holy L, Levine K, Kvaerner } \\
\text { G Norman. (2015) }\end{array}$ & $\begin{array}{l}\text { Se presentan las conclusiones deriva- } \\
\text { das del panel de especialistas en ORL } \\
\text { y se establece consenso acerca de la } \\
\text { DCFT. Fue redactado y revisado utilizan- } \\
\text { do un proceso interactivo que incluyó a } \\
\text { todos los miembros del panel. }\end{array}$ & $\begin{array}{l}\text { Consenso relativo a DCFT, definición } \\
\text { de DCFT, sus síntomas y signos, el rol } \\
\text { de pruebas objetivas y subjetivas en la } \\
\text { evaluación, diagnóstico diferencial de la } \\
\text { DCFT y finalmente se hacen recomenda- } \\
\text { ciones para investigaciones posteriores. }\end{array}$ \\
\hline $\begin{array}{l}\text { Responsiveness of the } \\
\text { 7-item Eustachian Tube } \\
\text { Dysfunction Question- } \\
\text { naire }\end{array}$ & $\begin{array}{l}\text { Van Roeyen S, Van de } \\
\text { Heyning P \& Van Rompaey } \\
\text { V. (2016) }\end{array}$ & $\begin{array}{l}\text { Se conformaron dos grupos, el GE } \\
\text { conformado por pacientes con DCFT } \\
\text { inducida por cambios de presión at- } \\
\text { mosférica y un GC conformado por } 22 \\
\text { pacientes sanos, con una edad prome- } \\
\text { dio de } 49 \text { y } 36 \text { años, respectivamente. } \\
\text { Al GE se le aplicó el EDTQ-7 antes y } \\
\text { después de ser sometidos a una in- } \\
\text { tervención mediante Tuboplastía de } \\
\text { Dilatación por Balón (TDB). Se evaluó } \\
\text { la capacidad de respuesta al cambio de } \\
\text { ETDQ-7 mediante coeficiente kappa de } \\
\text { Cohen. Los hallazgos de la evaluación } \\
\text { subjetiva se compararon con los pun- } \\
\text { tajes obtenidos mediante ETDQ-7 des- } \\
\text { pués de TDB. }\end{array}$ & $\begin{array}{l}\text { El ETDQ-7 puede discriminar entre pa- } \\
\text { cientes con DCFT inducida por cambios } \\
\text { de presión atmosférica y controles sa- } \\
\text { nos, y responde al cambio en pacientes } \\
\text { con DCFT inducida por cambios de pre- } \\
\text { sión atmostérica que han sido sometidos } \\
\text { a tuboplastía por dilatación de balón } \\
\text { (TDB). }\end{array}$ \\
\hline
\end{tabular}




\begin{tabular}{|c|c|c|c|}
\hline Título & Autor y fecha de publicación & Metodología & Principales resultados \\
\hline $\begin{array}{l}\text { Analysis of Eustachian } \\
\text { Tube Dysfunction by } \\
\text { Dynamic Slow Motion } \\
\text { Video Endoscopy and } \\
\text { Eustachian Tube Dys- } \\
\text { function Questionnaire in } \\
\text { Chronic Otitis Media }\end{array}$ & $\begin{array}{l}\text { Han WG, Yoo J, Rah YC, } \\
\text { Chang J, Im GJ, Song JJ, } \\
\text { Choi J. (2017) }\end{array}$ & $\begin{array}{l}\text { Estudio retrospectivo. Se evalúa la efec- } \\
\text { tividad de la endoscopía con video en } \\
\text { cámara lenta (EVCL) como herramienta } \\
\text { diagnóstica de la DCFT. } \\
\text { Además, se evalúa la correlación de la } \\
\text { EVCL con la maniobra de Valsalva, el } \\
\text { cuestionario ETDQ-7 y los hallazgos } \\
\text { intraoperatorios del CFT. } 46 \text { pacientes } \\
\text { conformaron la muestra. Se estudiaron } \\
46 \text { oídos con OMC y } 46 \text { oídos controles } \\
\text { sanos. }\end{array}$ & $\begin{array}{l}\text { Tanto la EVCL como el ETDQ-7 entregan } \\
\text { información del estado preoperatorio } \\
\text { del CFT. } \\
\text { En ETQQ-7, puntajes más altos se re- } \\
\text { lacionan con hallazgos de obstrucción } \\
\text { intraoperatoria del CFT. }\end{array}$ \\
\hline $\begin{array}{l}\text { Turkish validity and } \\
\text { reliability of Eustachian } \\
\text { Tube Dysfunction } \\
\text { Questionnaire-7 }\end{array}$ & $\begin{array}{l}\text { Özgür E, Bilgen C, Cengiz } \\
\text { Özyurt B. (2017) }\end{array}$ & $\begin{array}{l}\text { Se realizó la adaptación de la versión } \\
\text { original del ETDQ-7 al idioma turco. } \\
\text { Luego, se conformaron } 2 \text { grupos; } 40 \\
\text { pacientes con diagnóstico de DCFT en } \\
\text { el GE y } 40 \text { individuos sanos en el GC. Se } \\
\text { aplicó el ETDQ-7 a ambos grupos. Dos } \\
\text { semanas después de la primera evalua- } \\
\text { ción, } 15 \text { pacientes sin ninguna inter- } \\
\text { vención llenaron la escala nuevamente. }\end{array}$ & $\begin{array}{l}\text { El coeficiente alfa de Cronbach fue de } \\
0.714 \text {. El coeficiente de confiabilidad } \\
\text { test retest fue de } 0.792 \text {. En el grupo con } \\
\text { DCFT, los puntajes totales se encontra- } \\
\text { ron significativamente más altos que } \\
\text { en el grupo control. Se encontró que la } \\
\text { versión turca del ETDQ-7 fue altamente } \\
\text { válida y confiable. Se recomienda su uso } \\
\text { en el screening de la DCFT así como en la } \\
\text { evaluación de resultados de tratamiento. }\end{array}$ \\
\hline $\begin{array}{l}\text { The efficacy of the Eus- } \\
\text { tachian Tube Dysfunction } \\
\text { Questionnaire (ETDQ-7) } \\
\text { for patulous Eustachian } \\
\text { tube patient }\end{array}$ & $\begin{array}{l}\text { Ikeda R, Kikuchi T, Miyazaki } \\
\text { H, Hidaka H, Kawase T, Katori } \\
\text { Y \& Kobayashi T. (2017) }\end{array}$ & $\begin{array}{l}\text { Estudio prospectivo de registros mé- } \\
\text { dicos. Participaron } 36 \text { pacientes y } 47 \\
\text { oídos con DCFT-P; y } 15 \text { pacientes y } 15 \\
\text { oídos controles en GC. Se evaluó con el } \\
\text { ETDQ-7 y la Scale Patulous Eustachian } \\
\text { tube handicap inventory-10 (PHI-10). } \\
\text { Los pacientes con DCFT se dividieron } \\
\text { en dos grupos según la gravedad de los } \\
\text { síntomas utilizando la puntuación PHI- } \\
\text { 10. En los resultados, se observó que el } \\
\text { valor de Cronbach del grupo DCFT-P, el } \\
\text { cual fue de } 0,765 \text {. }\end{array}$ & $\begin{array}{l}\text { La puntuación total promedio de ETDQ-7 } \\
\text { en el grupo control fue de } 7.6 \pm 1,1 \text { y } 22.5 \\
\pm 10,0 \text { en el grupo de DCFT-P. El puntaje } \\
\text { total promedio del ETDQ-7 en el grupo } \\
\text { de DCFT-P leve o moderado fue de } 19,9 \\
\pm 9.0 \text { y en el grupo de DCFT-P grave, fue } \\
\text { de } 25.3 \pm 11,1 \text {. } \\
\text { El ETDQ- } 7 \text {, siendo una herramienta útil } \\
\text { en el diagnóstico de la DCFT, no diferen- } \\
\text { cia entre DCFT-OC y DCFT-P, por tanto } \\
\text { los resultados del mismo deben ser } \\
\text { interpretados cuidadosamente en el con- } \\
\text { texto de una decisión quirúrgica. }\end{array}$ \\
\hline $\begin{array}{l}\text { Accuracy of the ETDQ-7 } \\
\text { for Identifying Persons } \\
\text { with Eustachian Tube } \\
\text { Dysfunction }\end{array}$ & $\begin{array}{l}\text { Teixeira M, Douglas J and } \\
\text { Alper C. (2017) }\end{array}$ & $\begin{array}{l}\text { Estudio transversal. Se evaluó la co- } \\
\text { rrelación entre el ETDQ-7 y los resul- } \\
\text { tados de la timpanometría, en cuanto } \\
\text { a porcentaje de presión equilibrada de } \\
\text { oído medio después de } 5 \text { degluciones } \\
\text { (Peq5), para membranas timpánicas } \\
\text { intactas, o mediante la prueba de } \\
\text { inflación-deflación, para membranas } \\
\text { timpánicas no intactas. } \\
\text { Como parámetro para definir la DCFT se } \\
\text { utilizaron valores en ETDQ-7 } \geq 14.5 \text { y en } \\
\text { timpanometría, PEq5 }<60 \% \text {. Se evaluó } \\
\text { sensibilidad, especificidad y ROC para } \\
\text { la correlación entre resultados obteni- } \\
\text { dos en ETDQ-7 y la timpanometría. }\end{array}$ & $\begin{array}{l}\text { El puntaje ETDQ-7 tuvo mayor corre- } \\
\text { lación con los síntomas asociados a la } \\
\text { DCFT que con resultados de la medida } \\
\text { objetiva utilizada para evaluar la funcio- } \\
\text { nalidad del CFT. }\end{array}$ \\
\hline $\begin{array}{l}\text { Translation, validation } \\
\text { and cultural adaptation } \\
\text { of "The Eustachian Tube } \\
\text { Dysfunction Question- } \\
\text { naire-7" (ETDQ-7) to } \\
\text { Brazilian Portuguese (BR) }\end{array}$ & $\begin{array}{l}\text { Pires F, Tsuneo E, lure F, } \\
\text { Barros F \& Gurgel G. (2017) }\end{array}$ & $\begin{array}{l}\text { Se adaptó y validó el cuestionario } \\
\text { ETDQ-7 original, al idioma portugués } \\
\text { de Brasil. En el estudio participaron } 50 \\
\text { pacientes, de los cuales } 20 \text { formaron } \\
\text { el grupo con DCFT-OC y } 30 \text { formaron } \\
\text { el GC. }\end{array}$ & $\begin{array}{l}\text { Los resultados obtenidos con la versión } \\
\text { brasileña del ETDQ-7 reproducen los ha- } \\
\text { llazgos obtenidos mediante versión origi- } \\
\text { nal del cuestionario en inglés. El punto de } \\
\text { corte para el diagnóstico de DCFT crónica } \\
\text { fue } \geq 14 \text {. El ETDQ-7 mostró una alta sensi- } \\
\text { bilidad y especificidad. Se recomienda su } \\
\text { uso como herramienta para el diagnósti- } \\
\text { co, seguimiento y manejo de la DCFT. }\end{array}$ \\
\hline
\end{tabular}




\begin{tabular}{|c|c|c|c|}
\hline Título & Autor y fecha de publicación & Metodología & Principales resultados \\
\hline $\begin{array}{l}\text { The Hebrew version of } \\
\text { the Eustachian tube dys- } \\
\text { function questionnaire- } 7\end{array}$ & $\begin{array}{l}\text { Omer J Ungar, Oren Cavel, } \\
\text { Gilad S Golan, Yahav Oron, } \\
\text { Oshri Wasserzug \& Ophir } \\
\text { Handzel (2018) }\end{array}$ & $\begin{array}{l}\text { Se validó la versión hebrea del ETDQ-7 } \\
\text { y se estudió la confiabilidad test retest. } \\
\text { Participaron } 115 \text { pacientes, todos ha- } \\
\text { blantes nativos del hebreo, con y sin } \\
\text { DCFT. }\end{array}$ & $\begin{array}{l}\text { El coeficiente alfa de Cronbach en test, } \\
\text { retest fue de } 0,918,0,922 \text { y } 0,890 \text {, res- } \\
\text { pectivamente. El área bajo la curva para } \\
\text { la primera y segunda aplicación de los } \\
\text { cuestionarios fue } 0,956 \text {, representando } \\
\text { una excelente validez discriminante. } \\
\text { El ETDQ-7 en su versión hebrea, mos- } \\
\text { tró consistencia interna significativa y } \\
\text { relevancia clinica. Puede ser utilizada en } \\
\text { evaluación para el diagnóstico y manejo } \\
\text { de la DCFT. }\end{array}$ \\
\hline $\begin{array}{l}\text { The Performance of } \\
\text { Patient-reported Outcome } \\
\text { Measures as Diagnostic } \\
\text { Tools for Eustachian Tube } \\
\text { Dysfunction }\end{array}$ & $\begin{array}{l}\text { Smith M, Cochrane I, } \\
\text { Donnelly N, Axon P, Tysome } \\
\text { J. (2018) }\end{array}$ & $\begin{array}{l}\text { El estudio desarrolló y validó una nue- } \\
\text { va herramienta de autoevaluación de } \\
\text { síntomas: el Cambridge Eustachian } \\
\text { Tube Dysfunction Assessment (CET- } \\
\text { DA), para distinguir entre una DCFT-P } \\
\text { de una DCFT-OC. Se compararon los } \\
\text { resultados, con los obtenidos de la apli- } \\
\text { cación ETDQ-7 y a su vez con pruebas } \\
\text { objetivas de sonotubometría y tuboma- } \\
\text { nometría. Se determinó, la precisión de } \\
\text { ambos cuestionarios en la detección } \\
\text { de la DCFT. El estudio incluyó } 7 \text { casos } \\
\text { con DCFT-P, } 60 \text { con DCFT-OC, } 33 \text { con- } \\
\text { troles sanos y } 24 \text { controles con sínto- } \\
\text { mas derivados de pérdida de audición } \\
\text { o enfermedad de Mèniére. Se evaluó la } \\
\text { consistencia interna, efecto de techo y } \\
\text { piso, confiabilidad test- retest, validez } \\
\text { estructural y de criterio de los cuestio- } \\
\text { narios de autovaloración de síntomas } \\
\text { (CETDA y ETDQ-7). }\end{array}$ & $\begin{array}{l}\text { El ETDQ-7 es un instrumento válido para } \\
\text { evaluar la función del CFT, por lo tanto } \\
\text { es utilizado como prueba estándar en la } \\
\text { evaluación del nuevo cuestionario pro- } \\
\text { puesto. } \\
\text { El índice de correlación entre CETDA y } \\
\text { ETDQ-7 fue de } 0,89 \text {, ambas con exce- } \\
\text { lente repetibilidad. El ETDQ-7 es una } \\
\text { herramienta válida para el diagnóstico de } \\
\text { la DCFT en sujetos sin otra patología au- } \\
\text { ditivay no permitiría identificar la DCFT-P } \\
\text { de la DCFT-OC. }\end{array}$ \\
\hline
\end{tabular}

y Mèniére ${ }^{28}$, en los que se señala que el ETDQ-7 no sería recomendado para la evaluación de la DCFT en pacientes que presentaran otras enfermedades 0 alteraciones de oído.

Respecto al análisis de la consistencia interna, varios fueron los estudios que miden esta variable. En 2017, Özgür y cols informaron un alfa de cronbach de 0,714 para todo el cuestionario ${ }^{25}$. Por otra parte, el trabajo de Omer y cols en 2018, aplicó tres veces el cuestionario; antes y después de una intervención médica. Obteniendo un puntaje de 0,918 en la primera hora posterior a la intervención médica y de 0,922 en la segunda hora posterior; una semana después de la primera aplicación obtuvo un puntaje de 0,890 en la consistencia interna. En los tres estudios mencionados anteriormente, se exhiben buenos resultados para el ETDQ-7 en cuanto al coeficiente de fiabilidad, presentándolo como una herramienta confiable. En cuanto al análisis cuantitativo y criterios de cor- te del ETDQ-7 en DCFT, Schröder y cols en 2013, reportaron una puntuación media de 24,7 para los sujetos que padecen DCFT; hallazgos que se correlacionan con lo observado por lkeda y cols, en 2017, quienes obtuvieron una media de $22,5 \pm 10,0$ puntos, tanto en pacientes con DCFT- OC, como por DCFT-P. En Corea del Sur, se demostró que las personas que presentan rinitis alérgica obtienen puntajes más altos en el ETDQ-7 que las que no presentan dicha patología ${ }^{5}$ y se reporta finalmente un criterio de corte de 14 puntos para la presencia de DCFT-OC ${ }^{26}$.

\section{DISCUSIÓN}

EI ETDQ-7 fue diseñado para ser aplicado en sujetos con síntomas de DCFT, sin otra patología otológica. Esto explicaría la disminución en su especificidad cuando se aplica en poblaciones con otra patología 
de oído adicional, lo que no ha sido aconsejado por Ios autores del cuestionario original. De acuerdo con lo reportado ${ }^{17,26,27}$ el ETDQ-7 es una herramienta útil para comparar rendimientos entre evaluaciones pre y posoperatorias y se puede utilizar como una herramienta adicional para el seguimiento después de un tratamiento quirúrgico. Así mismo, se plantea que el cuestionario permite monitorear las implicancias de la DCFT y su respuesta al tratamiento ${ }^{28}$. Asimismo, sus limitaciones en diagnóstico diferencial entre DCFT-OC y DCFT-P no son exclusivas, siendo una condición común, tanto de las diferentes medidas de resultados informadas por el paciente, como de otros métodos objetivos utilizados como herramienta estándar para el diagnóstico de la DCFT, como es el caso de la impedanciometría ${ }^{28}$. Lo anterior orienta hacia que dicha limitación no corresponde a una característica privativa del ETDQ-7, sino a una dificultad transversal evidenciada en el diagnóstico diferencial de ambas etiologías. Por otra parte, las pruebas objetivas reportadas como idóneas para establecer el diagnóstico diferencial entre DCFT-OC y DCFT-P, la endoscopía nasal y la resonancia magnética, son de difícil acceso y elevado costo $2,4,12,9,10$.

Uno de los hallazgos interesantes de la presente revisión, es el consenso para la DCFT establecido mediante foro internacional de científicos y médicos especialistas en otorrinolaringología ${ }^{11}$. En él, se establece un marco referencial en cuanto a la definición, presentación clínica y diagnóstico de la DCFT, reportándose al ETDQ-7 como la única pauta o cuestionario de resultados informados por el paciente que ha sido sometida a estudios iniciales de validación ${ }^{11}$, siendo una herramienta que puede ayudar, tanto a establecer el diagnóstico, como en los procesos posteriores de intervención en pacientes con DCFT.

En resumen, los diversos indicadores estadísticos reportados en los estudios que han sido objeto de esta presente revisión demuestran que el ETDQ-7 es un instrumento válido y sensible para la detección de la DCFT. No se encontraron estudios de validación del cuestionario en la población chilena. Considerando la prevalencia de la DCFT en la población chilena, tanto infantil como adulta ${ }^{1}$, junto a las posibles complicaciones asociadas a dicho cuadro, se hace necesario generar investigaciones en esta línea, lo que se espera pueda contribuir a la práctica clínica, en beneficio directo de los usuarios que padecen una DCFT.

\section{CONCLUSIONES}

El presente estudio se centra en la utilidad del ETDQ-7 en el diagnóstico de la disfunción del conducto faringotimpánico (DCFT), planteando como pregunta central: ¿Qué información existe en publicaciones científicas, a nivel nacional e internacional, respecto al uso del ETDQ-7 como herramienta para el diagnóstico clínico de la disfunción del conducto faringotimpánico? A partir de los datos recopilados y respecto a la pregunta planteada, se concluye que existen investigaciones referidas a la evaluación y diagnóstico complementario de la DFCT mediante el uso del Eustachian Tube Dysfunction Questionnaire -7 ítems (ETDQ7), identificando a este cuestionario como un instrumento válido y confiable para su uso en el ámbito clínico. Como cuestionario autorreportado, el ETDQ-7 ofrece una perspectiva general de la severidad de una posible DCFT, siendo de fácil aplicación, rápido y sencillo de responder. Además, ha demostrado poseer buenos indicadores estadísticos, con un alto grado de validez, confiabilidad y consistencia interna ${ }^{24-26}$.

El ETDQ-7 es utilizado actualmente en diversos países para la detección de la DCFT, disminuyendo costos y tiempos de evaluación asociados. Esto permite optimizar el proceso diagnóstico de la DCFT al considerar y relevar los síntomas referidos por paciente. En cuanto a las críticas realizadas al ETDQ7 , la principal se refiere a la baja sensibilidad para el diagnóstico diferencial entre DCFT-OC y DCFT-P; sin embargo, los autores también concluyen que ninguna de las medidas de resultados informadas por el paciente tendría dicha capacidad de resolución entre ambas etiologías, por lo que el ETDQ-7 sería una herramienta útil para detectar de forma genérica la presencia de la DCFT, apoyando el diagnóstico clínico y una vez detectada la disfunción, se requeriría de métodos objetivos para realizar el diagnóstico diferencial entre las dos etiologías conocidas, la disfunción obstructiva y la de tipo patulosa ${ }^{28}$.

Si bien, ciertos métodos se han presentado como idóneos para realizar el diagnóstico certero y diferencial de la DCFT reconociendo claramente 
su etiología12, dichos métodos objetivos referidos son de elevado valor y sofisticación, por lo que no constituyen herramientas de acceso universal en salud pública². Como tarea para futuras investigaciones se plantea el optimizar la capacidad del cuestionario para discriminar entre las diferentes etiologías de DCFT, con miras a mejorar la versión de los 7 ítemes. Por último, la presente revisión

\section{BIBLIOGRAFÍA}

1. Hoffman H. Disfunción tubaria y sus complicaciones. Otorrinolaringología ISSN 1667- 8982. Suplemento de Salud(i) Ciencia 2012; 7: 2-10.

2. Pensak M, Choo D. Clinical Otology. 2015. New York: Thieme.

3. Salesa E, Bonavida A. Anatomía y fisiología del oído. En Tratado de audiología. Barcelona: Elsevier Masson. 2013; 1-9.

4. González L. Funcionalidad de la trompa de Eustaquio. Revista Gastrohnup, 2011; 13: S13-S17.

5. Han WG, Yoo J, Rah YC, Chang J, Im GJ, Song JJ, $\mathrm{CHOl} \mathrm{J}$. Analysis of Eustachian tube dysfunction by dynamic slow motion, video endoscopy and eustachian tube dysfunction Questionnaire in chronic otitis media. Clin Exp Otorhinolayngol 2017; 10: 315-20. http://doi.org/10.21053/ ceo.2016.01683.

6. HolmQuist J. Eustachian Tube Function Assessed with Tympanometry: A New Testing Procedure in Ears with Intact Tympanic Membrane. Acta Otolaryngol 1969; 68: 501-8. doi: 10.3109/00016486909121590.

7. Holmquist J. Eustachian Tube Function in Patients With Ear Drum Perforations Following Chronic Otitis Media: Results of a Simplified Testing Procedure by Deflation and Aspiration Methods. Acta Otolaryngol 1969; 68, 391-401.

8. Honjo I, Kumazawa T, Honda K. Simple Impedance Test for Eustachian Tube Function. Arch Otolaryngol 1981; 107: 221-3. doi:10.1001/ archotol.1981.00790400023.

9. Katz J, Chasin M, English K, Hood L and Tillery K. Handbook of Clinical Audiology, 2015; 7th ed. Buffalo, pp.137-63.

10. Finkelstein $A$, Caro J. Actualización en disfunción tubaria: Rol de la endoscopía nasal en su de la bibliografía aporta información preliminar relevante que justifica futuras investigaciones en el área, especialmente en latinoamérica, puesto que existe escasa información y evidencia científica en el tema, por lo que una de las posibles proyecciones del presente estudio es desarrollar estudios de adaptación y validación del ETDQ-7 en nuestro país.

evaluación y tratamiento. Rev Otorrinolaringol Cir Cabeza Cuello 2008; 68: 59-66.

11. Schilder A, M Bhutta, C Butler, C Holy, L Levine, K Kvaerner, G Norman. Eustachian tube dysfunction: consensus statement on definition, types, clinical presentation and diagnosis. Clin Otolaryngol 2015; 40: 407-11.

12. Aedo C, Muñoz D, Der C. Trompa patulosa. Rev Otorrinolaringol Cir Cabeza Cuello 2009; 69: 61-70. https://dx.doi.org/10.4067/S071848162009000100012.

13. McCoul E, Anand V, Christos P. Validating the Clinical Assessment of Eusatachian Tube Dysfunction: The Eustachian Tube Dysfunction Questionnaire (ETDQ-7). Laryngoscope 2012; 122: $1137-41$.

14. Smith M, Scoffings D, Tysome J. Imaging of the Eustachian tube and its function: a systematic review. Neuroradiology 2016; 58: 543-56.

15. Sudhoff H, Mueller S. Treatment of pharyngotympanic tube dysfunction. Auris Nasus Larynx 2018; 45: 207-14.

16. Correa A. Manual de Otorrinolaringología 1999, Chile: Mediterráneo.

17. Schroeder S, Lehmann M, Sauzet 0, Ebmeyer J, Sudhoff H. A novel diagnostic tool for chronic obstructive Eustachian Tube Dysfunction- The Eustachian Tube Score. Laryngoscope 2014; 125: 703-8.

18. Smith M, Tysome J. Análisis de la función de la trompa de Eustaquio: una revisión. Clin Otolaryngol 2015; 40: 300-11.

19. Teixeira M, Douglas J, Alper C. Accuracy of the ETDQ-7 for Identifying Persons with Eustachian Tube Dysfunction. Otolaryngol Head Neck Surg 2018; 158: 83-9.

20. Prieto L, Badia X. Cuestionario de salud: Concepto y metodología. Atención primaria 2001; 28: 3. 
21. López S, Morales AJM. ¿Para qué se administran las escalas, cuestionarios, tests e índices? Index de Enfermería 2005; 14: 48-9.

22. Molinero L. Cuestionarios de Salud. Asociación de la Sociedad Española de Hipertensión Liga Española para la lucha contra la hipertensión arterial. 1998. https://www.alceingenieria.net/ bioestadistica/cucadevi.pdf.

23. IKEDA R, KIKUCHI T, Miyazakı H, Hidaka H, Kawase T, KatoRI $Y$ \& Kobayashi T. The efficacy of th Eustachian Tube Dysfunction Questionnairre (ETDQ-7) for patulous Eustachian tube patient. Acta Otolaryngol 2017; 138: 6-9.

24. Omer J Ungar, Oren Cavel, Gilad S, Yahav Oron, Oshri Wasserzug, Ophir Handzel. The Hebrew version of the Eustachian tube dysfunction questionnaire-7, Hearing, Balance and Communication 2018; 16: 2, 114-9.

25. Özgür E, Bilgen C, Cengiz Özyurt B. Turkish validity and reliability of Eustachian Tube Dysfunction Questionnaire-7. Braz J Otorhinolaryngol 2018; 84: 435-40. doi: 10.1016/j.bjorl.2017.05.001.

26. Pires F, Tsuneo E, lure F, Barros F, Gurgel G.
Traducción, validación y adaptación cultural del "Cuestionario de disfunción del tubo de Eustaquio-7" (ETDQ-7) al portugués brasileño (BR). Revista Brasileña de Otorrinolaringologia 2017; 649: 1-9.

27. Schröder S, Lehmann M, Sudhoff H, Ebmeyer J. Beurteilung der chronisch- obstruktiven Tubenfunktionsstörung, evaluation des "Eustachian Tube Dysfunction Questionnaire" in der deutschen Übersetzung. HNO Journal 2013; 62: 162-4.

28. Smith M, Cochrane I, Donnelly N, Axon P, Tysome J. The Performance of Patient- reported Outcome Measures as Diagnostic Tools for Eustachian Tube Dysfunction. Otol Neurotol 2018; 39: 1-9. 29. Van Roeyen S, Van de Heyning P, Van Rompaey V. Value and Discriminative Power of the SevenItem Eustachian Tube Dysfunction Questionnaire. Laryngoscope 2015; 125: 2553-6. doi: 10.1002/ lary.25316.

30. Van Roeyen S, Van de Heyning P, Van Rompaey V. Responsiveness of the 7-item Eustachian Tube Dysfunction Questionnaire. J Int Adv Otol2016; 12: 106-8. 